\title{
VARIACIÓN ESPACIO-TEMPORALDE LAS POBLACIONES DE CRUSTÁCEOS, LARVAS DE POLIQUETOS Y PROTOZOOS DE LA LAGUNA MEROMÍCTICA ESTANY DE CULLERA (VALENCIA)
}

\author{
Rafael Oltra ${ }^{1}$ y Maria Rosa Miracle ${ }^{2}$ \\ Departament de Microbiologia i Ecologia, Facultat de Ciències Biològiques, Universitat de València, Dr. Moliner \\ 50,46100 Burjassot (Valencia). 'e-mail: rafael.oltra@uv.es, ${ }^{2}$ rosa.miracle@uv.es
}

\begin{abstract}
RESUMEN
En el plancton del Estany de Cullera se han reconocido I2 especies de copépodos y 6 cladóceros y además han aparecido ostrácodos, larvas, cirripedos de poliqueto, nemátodos y protozoos. En Cpoca estival las especies más importantes eran el copépodo Acanthocyclops robustus y el claddcero Moina micrura. Ambas se distribuian entre la superficie y la haloclina. En otoño el copépodo dominante era Calanipeda aquae-dulcis, que se situaba en las capas de la haloclina. También eran abundantes en la haloclina las larvas del poliqueto Ficopotamus enigmaticus y los ciliados Euplotes sp. En invierno la densidad planctónica disminuía notablemente y, coincidiendo con la mayor influencia marina, aparecían copépodos marinos como Acartia clausi, Acartia grani y Oithona nana. En primavera la especie caracteristica era Metacyclops minutus que se situaba en las capas superficiales, mientras que en la haloclina volvian a aparecer en densidades importantes $F$. enigmaticus y Euplotes sp. Las mayores densidades se dieron en verano y otoiio y las menores en invierno. Copépodos y cladóceros alcanzaron densidades máximas de $227 \cdot 10^{3} \mathrm{ind} / \mathrm{m}^{3}$ y $30 \cdot 10^{3} \mathrm{ind} / \mathrm{m}^{3}$ en verano y otoiio, respectivamente. Estas densidades equivalen a biomasas de 4.2 y $1.2 \mathrm{~g} / \mathrm{m}^{3}$ (peso fresco). Protozoos y poliquetos llegaron a densidades de $631 \cdot 10^{3} \mathrm{ind} / \mathrm{m}^{3}$ y $90 \cdot 10^{3} \mathrm{ind} / \mathrm{m}^{3} \mathrm{en}$ otoiio. Su contribución a la biomasa fue inferior a la de los crustaceos, alcanzando la cifra maxima de $39 \mathrm{mg} / \mathrm{m}^{3}$ en el caso de los protozoos y $876 \mathrm{mg} / \mathrm{m}^{3}$ en los poliquetos.
\end{abstract}

Palabras clave: salinidad, haloclina, distribución espacio-temporal, densidad, biomasa, copépodos, cladóceros, poliquetos, ciliados.

\begin{abstract}
Among the planktonfound in the Estany de Cullera lagoon were 12 species of copepods and 6 of cladocerans. There were also identified ostracoda, cirripeda, polychaete larvae, nematodes and protozoans. In Summer, the main species were the copepod Acanthocyclops robustus and the cladoceran Moina micrura. Both were present mainly between the surface and the halocline. In Autumn, the main copepod was Calanipeda aquae-dulcis, which wasfound in the layers of the halocline. The larvae of the polichaete Ficopotamus enigmaticus and the ciliates Euplotes sp. were also common in the halocline. In Winter, the density of plankton decreased notably. Marine copepods such as Acartia clausi, Acartia grani and Oithona nana then appeared, coinciding with the greatest marine influence. In Spring, the characteristic species was Metacyclops minutus, which wasfound in the top layers, whereas in the halocline, F. enigmaticus and Euplotes sp. reappeared in important numbers. Peak abundance occurred in Summer and Autumn and the lowest numbers in Winter. Copepods and cladocerans reached maximum densities of 227.10' ind $/ \mathrm{m}^{3}$ and $30 \cdot 10^{3} \mathrm{ind} / \mathrm{m}^{3}$ in Summer and Autumn, respectively. These densities are the equivalent of 4.2 and $1.2 \mathrm{~g} / \mathrm{m}^{3}$ biomass (fresh weight). Protozoans and polichaetes reached densities of $631.10^{\prime}$ ind $/ \mathrm{m}^{3}$ and $90.10^{3}$ ind/ $\mathrm{m}^{3}$ in Autumn. Their contribution to biomass was less than that of crustaceans, reaching maxima of $39 \mathrm{mg} / \mathrm{m}^{3}$ for the protozoa and $876 \mathrm{mg} / \mathrm{m}^{3}$ for the polichaetes.
\end{abstract}

Key words: salinity, halocline, spatio-temporal distribution, density, biomass, copepods, cladocerans, polichaetes, ciliates. 


\section{INTRODUCCIÓN}

Las lagunas litorales, que presentan condiciones fluctuantes con claros periodos de influencia marina alternando con otros de influencia continental y que además se estructuran verticalmente y horizontalmente, formando en muchos casos claras interfases con gradientes de temperatura, salinidad y oxigeno muy importantes, pueden ilustrar de manera simple los patrones distribucionales del zooplancton y en particular de los crustáceos. Los crustaceos son los componentes de mayor tamaiio y longevidad del zooplancton de agua dulce y constituyen normalmente la mayor proporción de su biomasa. Si bien los cladóceros tienen sucesiones estacionales marcadas, la distribución de los copkpodos es más dificil de interpretar por su ciclo de vida largo y complejo y por su mayor interiorización de la fluctuaciones estacionales. En cambio se pueden ver patrones claros en las lagunas litorales. Las distribuciones en las lagunas en las que domina un ciclo anual continental con una fauna de agua dulce no estan todavia sistematizadas, ya que aunque hay numerosos datos dispersos de recolecciones o listas faunisticas, existen pocas contribuciones que describan ciclos anuales o distribuciones en gradientes espaciales determinados. En lagunas de este tipo del Mediterráneo occidental existen ejemplos de ciclos anuales de microcrustaceos en la Camarga (Champeau, 1970), la Albufera de Menorca (Pretus, 1989), las lagunas del Delta del Ebro (Forés et al., 1986; Menéndez \& Comin, 1986), en el Parque Nacional Albufera de Valencia (Oltra \& Miracle, 1992; Alfonso \& Miracle, 1990, 1996) y las Albuferas de Adra (Carrillo et al., 1987). Sin embargo en ninguna de estas lagunas la influencia marina lleva a la formación de una destacada cuña de agua salada en el fondo, como ocurria el el Estany de Cullera a principios de la década de los 80 , cuando todavia tenia una barra bien formada que la separaba del mar (Miracle \& Vicente, 1985, Oltra \& Miracle a, este volumen). En este trabajo se describe la dinamica de las poblaciones principalmente de crustaceos en la laguna de Cullera en aquella kpoca. Se hace luego un estudio de los otros gru- pos que ocurren en el zooplancton, como son las larvas de poliqueto y protozoos y luego se estudian las proporciones de los diferentes grupos incluyendo el total de rotiferos. La descripción detallada de las distribuciones de las distintas especies de rotiferos se recoge en Oltra y Miracle (2000a) publicado en este mismo volumen. En esta laguna, al igual que en otras lagunas con variable influencia marina (Menkdez \& Comin, 1986; Pretus et al., 1992), la entrada periodica de agua de mar hacia que en el plancton estuvieran presentes especies eurihalinas de procedencia continental y especies marinas, siendo uno de los objetivos principales del presente trabajo el estudio del condicionamiento de las distribuciones de estas especies al gradiente de salinidad. Este estudio, junto con el realizado simultaneamente sobre los rotiferos (Oltra y Miracle, 2000a) y el basado en la elaboración conjunta de los datos (Oltra y Miracle, 2000b) pretende dar una visión del zooplancton de esta laguna cuando todavia conservaba su condición meromictica original. La laguna desapareció como tal al final de la década de los años ochenta debido a obras de ampliación en el canal que la comunicaba con el mar y quedo transformada en un estuario.

\section{MATERIAL Y MÉTODOS}

Una descripción del Estany de Cullera puede verse en Oltra \& Miracle (2000a), en este volumen y en otros trabajos (Miracle \& Vicente, 1983; Rojo \& Miracle, 1984, 1989, Rojo et al., 1986) sobre el fitoplancton, producción primaria y bacterias fotosintéticas derivados de las muestras y datos recogidos simultáneamente en las mismas campañas de muestreo del presente estudio. En la laguna, de aproximadamente $2000 \mathrm{~m}$ y anchura máxima de $150 \mathrm{~m}$, se establecieron 3 estaciones de muestreo: (1) cerca del canal que la comunicaba con el mar, con una profundidad de $4.5 \mathrm{~m}$; (2) en la parte central, con una profundidad de $7.5 \mathrm{~m}$; (3), cerca del extremo interior, con una profundidad de $3.5 \mathrm{~m}$. $\mathrm{La}$ cuña de agua salada del fondo afectaba más a las estaciones 1 y 2 que a la 3 . La haloclina se aproximaba a $1 \mathrm{~m}$ de la superficie en los meses fnos, 
debido a la mayor entrada de agua de mar, y descendia hasta $3.5 \mathrm{~m}$ en los meses cálidos en los que predominaba la influencia continental y el lavado ejercido por el agua oligohalina superficial. Las condiciones anóxicas del fondo desaparecían temporalmente en los meses fríos en la estación 1 , pero no asi en la estación 2 en la que la oxiclina siempre estuvo presente entre $3.5 \mathrm{~m}$ (verano) y 4.5 $\mathrm{m}$ de profundidad (invierno). En la estacion 3 se daban condiciones anoxicas solo ocasionalmente al final del periodo estival (Oltra, 1993; Oltra \& Miracle, 2000a).

En el periodo de estudio, que comprendió desde agosto de 1980 hasta octubre de 1981 , se realizaron 9 muestreos en los que se tomaron muestras en el perfil vertical en las tres estaciones de muestreo. En la estacidn 2 además se efectuaron muestreos a diferentes horas del ciclo diario y se realizaron tres muestreos adicionales durante julio-octubre de 1982. Las muestras fueron recogidas con una doble botella Van Dorn de 81 de capacidad o con una botella Ruttner de 2.61 de capacidad, filtrando 7.8, 8.0 o 10.41 de agua in situ con mallas nytal de $45 \mu \mathrm{m}$ y se fijaban con formalina al $4 \%$. Las especies zooplanctónicas fueron recontadas con la ayuda de microscopios invertidos a 100 y 200 aumentos. Se utilizaron las monografias de Dussart (1967, 1969), Kiefer, (1978) para la identificacidn de copépodos, las de Flossner (1972), Alonso (1985), para la identificación de cladóceros, las de Kudo (1976), Corliss (1979), Curds (1982) para la identificacidn de protozoos y la de Bianchi (1981) para la identificación de poliquetos.

El número medio de individuos por unidad de volumen en la columna de agua, se calculó de la forma descrita en Oltra y Miracle (2000a). La biomasa (peso fresco) se calculó a partir del biovolumen y considerando que la densidad del zooplancton es 1. Se ha aplicado las expresiones de Edmonson (1971) para el calculo de biovolumen de copépodos y cladóceros, se ha considerado a las larvas del poliqueto Ficopotamus enigmaticus como cilindros para el mismo calculo y se han aplicado las expresiones de Ruttner-Kolisko (1977): $\mathrm{v}=0.02 \mathrm{a}^{3}$ (medio cono) para el calculo del biovolumen de los tintinidos y $\mathrm{v}=0.08 \mathrm{a}^{3}$ (cilindro bajo) para el calculo del biovolumen de los restantes protozoos.

\section{RESULTADOS}

En el plancton del Estany se han reconocido 12 especies de copépodos, 6 de cladóceros, una de larva de poliqueto y, además, ostrácodos, nauplios de cirrípedos, nemátodos y cuatro taxones de protozoos. En la Tabla 1 se enumeran y se indica el valor de algunos parámetros ambientales para estas especies durante su periodo de aparición o en el momento de máxima abundancia. Las especies más frecuentes y abundantes han sido los copépodos Calanipeda aquae-dulcis, Acunthocyclops robustus, Metacyclops minutus, el claddcero Moina micruru, larvas del poliqueto Ficopotumus enigmaticus y ciliados del gknero Euplotes.

\section{Copépodos}

- Culanipeda aquae-dulcis fue una de las pocas especies perennes en el plancton. A lo largo del ciclo anual se encontró con mayor abundancia (310 ind/l) en otoño y verano y mas en las estaciones 1 y 2 que en la 3 (Fig. I). Se ha encontrado a conductividades desde 1.1 hasta $52.7 \mathrm{mS} / \mathrm{cm}$ y temperaturas de 8 a $30^{\circ} \mathrm{C}, 10$ cual confirma el carácter eurihalino y euritermo de la especie. En el perfil vertical se situaba preferentemente sobre la haloclina. La poblacidn estuvo compuesta mayoritariamente por nauplios y sólo en las kpocas de maximos de poblacidn adquirieron los copepoditos y adultos densidades importantes (Fig. 1). La densidad fue minima entre los meses de noviembre de 1980 y febrero de 1981, coincidiendo con las temperaturas mínimas y un ascenso de la haloclina en febrero. En el mes de mayo de 1981 se produjo un crecimiento de la poblacidn que fue mas acusado en las estaciones 1 y 2 , en las cuales la densidad media se situó alrededor de 20 ind/l. AI final de junio de 1981, ya en época de estratificación, se alcanzo un primer rnaximo estival, con densidades de 100 ind/l en la estacion 1. Tras una ligera disminución de la densidad en septiembre de 1981, se produjo un fuerte crecimiento a mediados de octubre 
Tabla 1. Crustáceos, poliquetos, nemátodos y protozoos hallados en el plancton del Estany de Cullera. Se indica la estacidn del año y punto de muestreo en que han aparecido y en donde se han alcanzado mayor abundancia (subrayados), su densidad media cuando han estado presentes y rango de temperatura y conductividaden los momentos de mayor densidad o durante su periodo de aparición, en el caso de las especies poco abundantes $\left(^{*}\right)$. (P, primavera: $\mathrm{V}$, verano: O,otoño, I, invierno; Prn, perenne). Crustaceans, polichaetes, nematodes and protozoafound in the plankton of the Estany de Cullera lagoon. Thefollowing data are indicated: season of the year and sampling point when and where the species occurred and when and where thay reached peak abundance (underlined),mean density when present, temperature and conductivity range during population peaks only or when ever present, in the case of the minor species (*). (Sp, Spring; S, Summer; A, Autumn, W, Winter; Prn, perennial).

\begin{tabular}{|c|c|c|c|c|c|c|}
\hline Especies & $\begin{array}{c}\text { Ocurrencia } \\
(\%)\end{array}$ & $\begin{array}{c}\text { Estación } \\
\text { del año }\end{array}$ & $\begin{array}{l}\text { Punto de } \\
\text { muestreo }\end{array}$ & $\begin{array}{l}\text { Densid. } \\
\text { (ind//) }\end{array}$ & $\begin{array}{c}\text { Temp. } \\
\left({ }^{\circ} \mathrm{C}\right)\end{array}$ & $\begin{array}{c}\text { Cond. } \\
(\mathrm{mS} / \mathrm{cm})\end{array}$ \\
\hline \multicolumn{7}{|l|}{ COPEPODOS } \\
\hline Calanipeda aquae-dulcis & 91.3 & $\operatorname{Prn}(\underline{O})$ & $1,2,3$ & 25.6 & 22 & $6.3-29.6$ \\
\hline Acanthocyclops robustus & 79.0 & $\underline{\mathrm{V}}, \mathrm{O}, \mathrm{I}$ & $1,2, \underline{3}$ & 22.3 & $22-26$ & $1.1-4.5$ \\
\hline Metacyclops minutus & 16.0 & $\underline{\mathrm{P}}$ & $1,2, \underline{3}$ & 6.7 & 21 & 1.8 \\
\hline Ergasillus sieboldi & 14.8 & $\operatorname{Prn}(\underline{Q})$ & $1,2, \overline{3}$ & 0.2 & 23 & 26 \\
\hline Halicyclops neglectus & 5.5 & $\mathrm{P}$ & $1,2,3$ & 0.4 & 16 & 32.1 \\
\hline Euterpina acutifrons* & 6.1 & $\mathrm{O}, \mathrm{I}, \mathrm{P}$ & $1,2,3$ & 0.2 & $13-20$ & $32.6-48.9$ \\
\hline Horsiella $s p *$ & 3.7 & $\mathrm{O}, \mathrm{I}, \mathrm{P}$ & $1,2,3$ & 0.5 & 15 & $32.6-49.6$ \\
\hline Harpacticus littoralis* & 0.6 & $\mathrm{O}$ & 1 & 0.1 & - & - \\
\hline Nitocrasp $p^{*}$ & 4.9 & $\mathrm{P}, \mathrm{V}, \mathrm{O}$ & 2 & 0.2 & $19-26$ & $1.7-2.6$ \\
\hline Acartia clausi* & 3.7 & $\mathrm{O}, \mathrm{I}$ & 2,3 & 0.2 & $14-20$ & $39.6-50.2$ \\
\hline Acartia grani* & 1.8 & $\mathrm{O}$ & 1,2 & 0.2 & $18-22$ & $29.6-47.6$ \\
\hline Oithona nana* & 8.6 & $\mathrm{O}, \mathrm{I}, \mathrm{P}$ & $1,2,3$ & 0.2 & $13-21$ & $32.1-50.2$ \\
\hline \multicolumn{7}{|l|}{ CLADÓCEROS } \\
\hline Moina micrura & 27.7 & V & $1,2,3$ & 7.8 & $26-30$ & $2-8.8$ \\
\hline Bosmina longirostris* & 2.4 & $\mathrm{P}, \mathrm{O}$ & $1,2,3$ & 0.2 & $18-22$ & $1.7-2$ \\
\hline Macrothrix hirsuticornis* & 1.8 & $\mathrm{P}$ & 2,3 & 0.2 & $20-22$ & $1.7-3.5$ \\
\hline Alona rectangula* & 1.8 & $\mathrm{P}$ & 2,3 & 0.2 & $20-22$ & $1.7-2$ \\
\hline Leydigia acanthocercoides* & 1.2 & $\mathrm{~V}$ & 2 & 0.2 & $22-26$ & $1.1-10.5$ \\
\hline Leydigia leydigi* & 0.6 & $\mathbf{V}$ & 2 & 0.1 & 22 & 18.9 \\
\hline OSTRÁCODOS* & 1.8 & $\mathrm{~V}, \mathrm{O}$ & 2,3 & 0.2 & 26 & 1.1 \\
\hline CIRRÍPEDOS (nauplios)* & 2.4 & $\mathrm{O}$ & 1,2 & 0.3 & $18-23$ & $26-47.6$ \\
\hline \multicolumn{7}{|l|}{ POLIQUETOS } \\
\hline Ficopotamus enigmaticus (larvas) & 42.5 & $\operatorname{Prn}(\underline{\mathrm{O}})$ & $1,2,3$ & 23.4 & 23 & $26-29$ \\
\hline NEMÁTODOS & 32.0 & $\operatorname{Prn}(\underline{\mathrm{O}})$ & $1,2, \underline{3}$ & 0.3 & 9 & 1.3 \\
\hline \multicolumn{7}{|l|}{ PROTOZOOS } \\
\hline Tintinnida & 3.0 & $\underline{I}$ & $1, \underline{2}, 3$ & 2.4 & 13 & 41.9 \\
\hline Euplotidae & 39.5 & $\operatorname{Prn}(\underline{\mathrm{O}})$ & $1,2,3$ & 79.4 & 21 & 9 \\
\hline Arcellidae & 33.9 & $\operatorname{Prn}(\underline{V})$ & $1,2, \underline{3}$ & 0.4 & 26 & 1.2 \\
\hline Halteriidae & 36.4 & $\operatorname{Prn}(\underline{\mathrm{V}})$ & $1,2, \overline{3}$ & 4.0 & 22 & 9 \\
\hline
\end{tabular}

estacion 2. La mayor densidad se registro en julio de 1982 (70 ind/l).

También fueron identificados los cladóceros Bosmina longirostris, Macrothrix hirsuticornis, Alona rectangula, Leydigia acanthocercoides y Leydigia leydigi. Estas especies han aparecido siempre con baja densidad $\mathbf{y}$, preferentemente, en primavera y verano, en las estaciones 2 y 3 , a salinidades bajas e inferiores, en general, a aquellas en que 10 hacen los copépodos (Tabla 1).

\section{Otros crustáceos}

Fueron encontrados escasos ejemplares del orden Ostracoda en muestras de verano y otoño 
siguiente, alcanzándose en las estaciones 1 y 2 densidades en torno a $200 \mathrm{ind} / 1$. Durante el verano y otofio de 1982 C. aquae-dulcis siguió una evolución semejante a la de aiios anteriores, es decir, una disminución de la población hacia finales de verano (agosto) y un nuevo aumento en octubre.

- Acanthocyclops robustus. La población estaba compuesta mayoritariamente por nauplios, habiéndose encontrado muy pocos copepoditos y adultos (Fig. 1). Estos últimos solo aparecieron en los meses estivales y generalmente en bajo numero, del orden de 1-2 por cada 10 litros. Unicamente en el mes de julio de 1982, en la estación 2, aumentó su presencia, observándose densidades de 5 a 10 ind/l. Este año present6 una pluviosidad mayor que los anteriores y la mayor afluencia de aguas dulces cargadas de nutrientes aumentó el nivel trófico. La especie apareció con mayor abundancia en la estación 3, menos influenciada por la intrusión marina que las estaciones 1 y 2 (Fig.2). Las mayores densidades, de 120 a 380 ind/l, se observaron en verano, con una temperatura de 22 a $26^{\circ} \mathrm{C}$

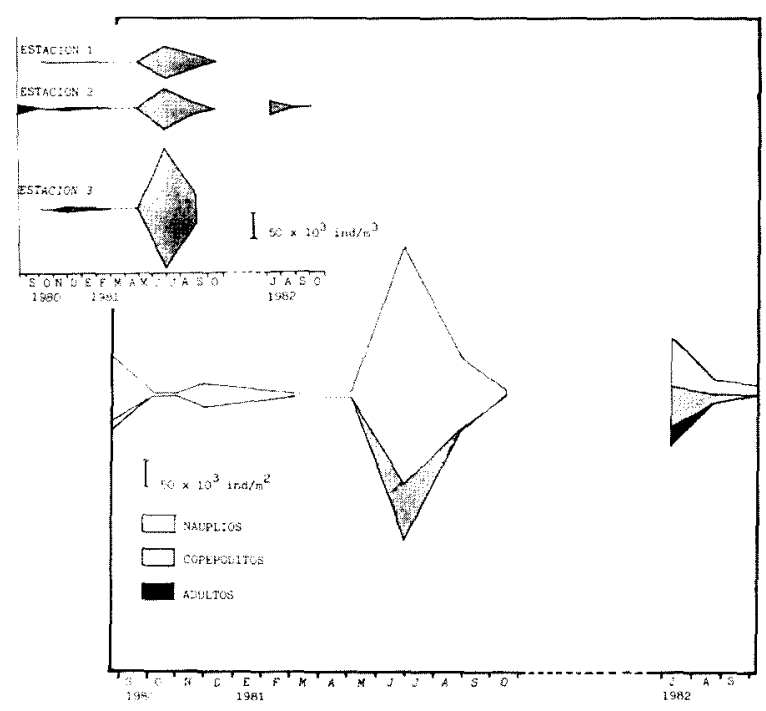

Figura 2. Dinámica de la poblacion de Acanthocyclops robustus en la estacion $2(\mathrm{ind} / \mathrm{m} 2)$. En la parte superior se ha representado la variación de la densidad media (ind/ $\mathrm{m} 3$ ) en las tres estaciones de rnuestreo. Population dynamics of Acanthocyclops robustus at station 2 (ind/m2). The top part shows mean density (ind/m3) at the three sampling stations. y conductividad de 1.1 a $4.5 \mathrm{mS} / \mathrm{cm}$. Por 10 tanto A. robustus, aunque ha sido hallada a conductividades muy variables, de 1.1 a $52.7 \mathrm{mS} / \mathrm{cm}$ (Tabla 1), parecia alcanzar sus mayores densidades a una conductividad inferior a la que 10 hacia el otro copépodo dominante, C. aquae-dulcis, y además presentaba sus maximos en verano, mientras que el calanoide 10 hacia en otofio.

- Metacyclops minutus. Unicamente apareció en el mes de mayo de 1981. La población estaba compuesta mayoritariamente por nauplios $y$, en menor proporción, por copepoditos, siendo muy raros los adultos. Result6 mas abundante en la estación 3, en donde alcanzó densidades de 10 a 25 ind/l (Fig.3). No se encontraron ejemplares en muestreos posteriores, porque es una especie de diapausa estival (Pont 1977), propia de ambientes temporales que se desarrolla en los arrozales cercanos en primavera (Alfonso, 1996).

Acompañando a las especies dominantes aparecieron otras, generalmente en muy bajas densidades, con poca frecuencia y en momentos puntuales del ciclo anual (Tabla 1). Destaca por su mayor ocurrencia Ergasillus sieboldi, cuyas hembras son ectoparasitos de peces de aguas dulces y salobres, siendo los machos, nauplios y copepoditos de vida libre (Fryer, 1978). En el Estany se ha encontrado a 10 largo de todo el ciclo anual pero siempre en baja densidad. Las especies Euterpina acutifrons, Acartia clausi, Acartia grani y Oithona nana son marinas. Aparecieron en otofio e invierno, cuando la entrada de agua de mar era más intensa y la salinidad más alta y 10 hicieron a densidades bajas, nunca superiores a $1 \mathrm{ind} / /$ (Tabla 1 ). Tambien se han encontrado ocasionalmente en el plancton especies de habitos bénticos o litorales como Halicyclops neglectus y Harpacticus littoralis y especies de los generos Horsiella y Nitocra, que son considerados como eurihalinos y propios de aguas salobres de salinidad variable (Margalef, 1953; Dussart, 1967). Aparecieron con más frecuencia en otoiio e invierno, cuando la salinidad del agua era mayor.

\section{Cladóceros}

- Moina micrura. Únicamente apareció en verano (Fig. 3), siendo más abundante en la 

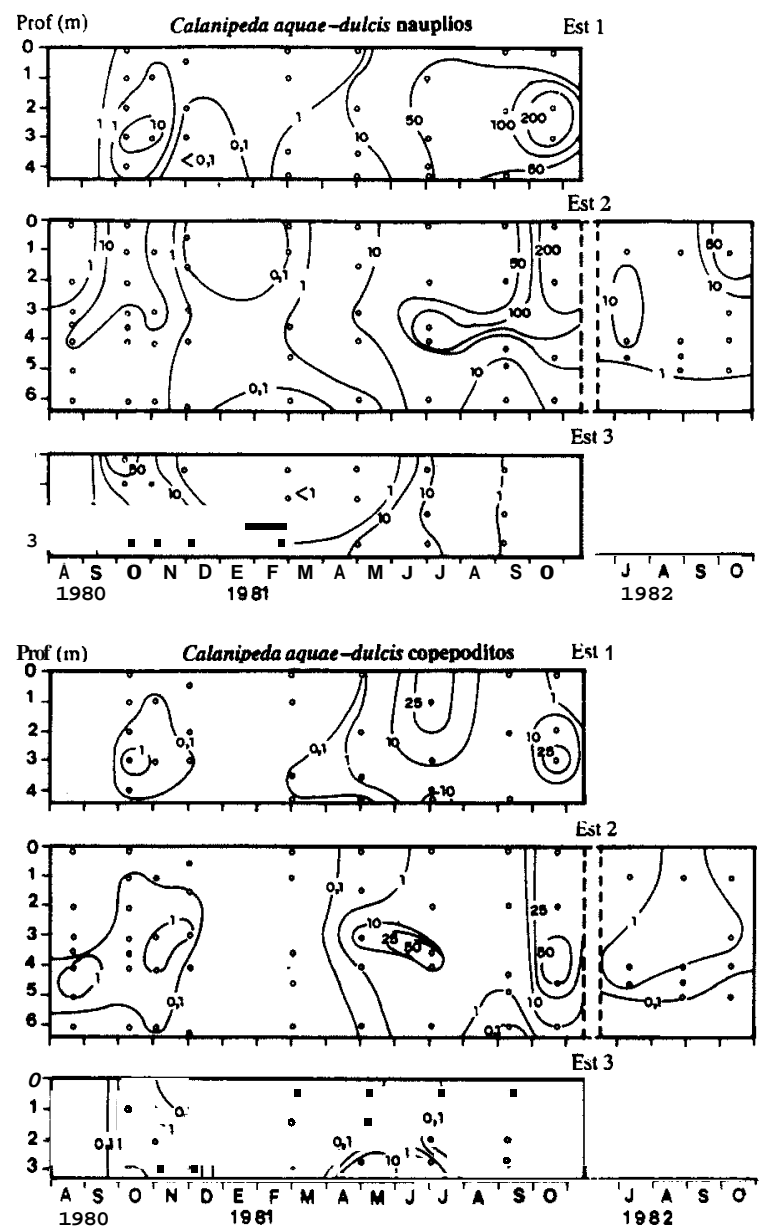

Prof $(\mathrm{m}) \quad$ Calanipeda aquae-dulcis adultos $\quad$ Est 1
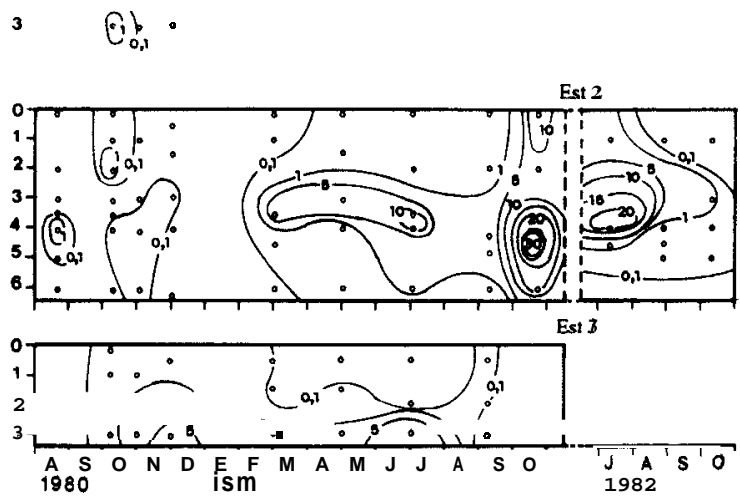
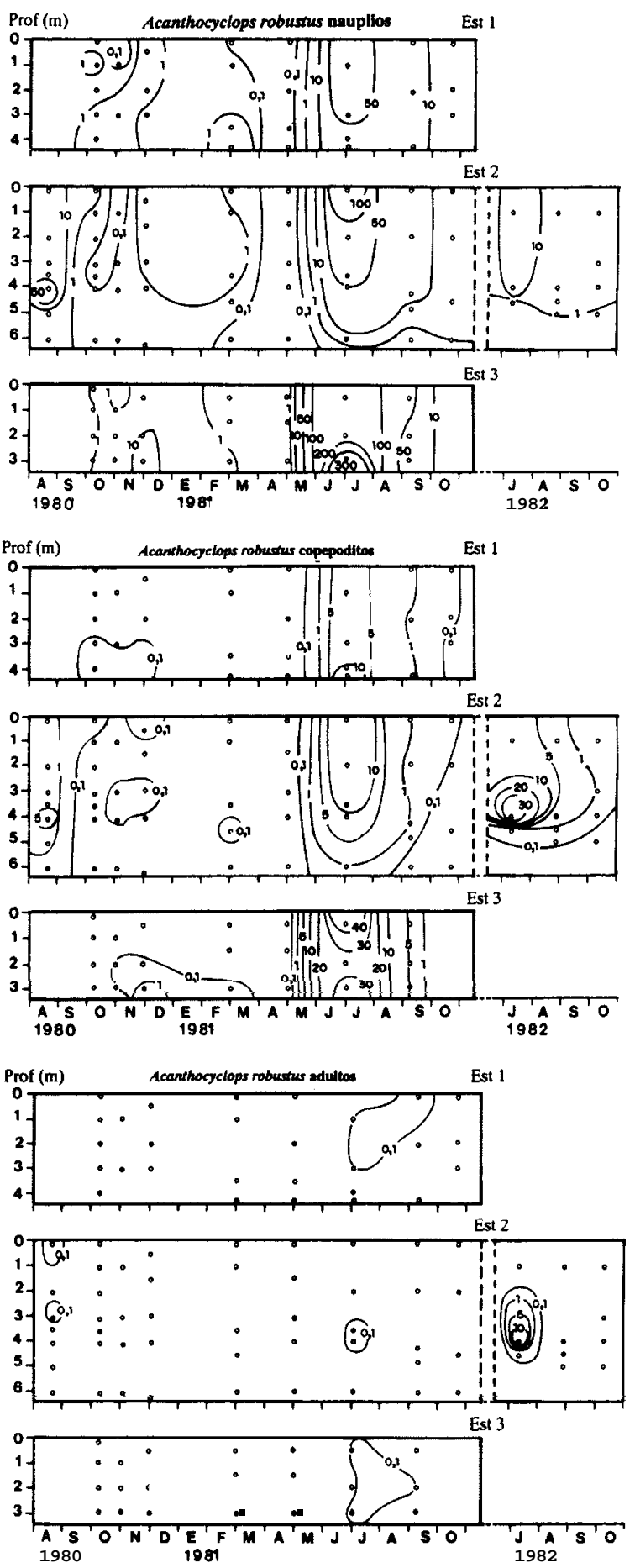

Figura 1. Distribución de la densidad de poblacion (ind/l) en función del tiempo y la profundidad en las tres estaciones de mustreo (Est. I, Est.2 y Est.3), de los nauplios, copepoditos y adultos de las especies Calanipeda aquae-dulcis (izquierda) y Acanthocyclops robustus (derecha). Seasonality and depth distribution (ind/l) of nauplii, copepodites and adults of Calanipeda aquae-dulcis (left) and Acanthocyclops robustus (right) in the three sampling points (Est.I, Est. 2 v Est.3). 

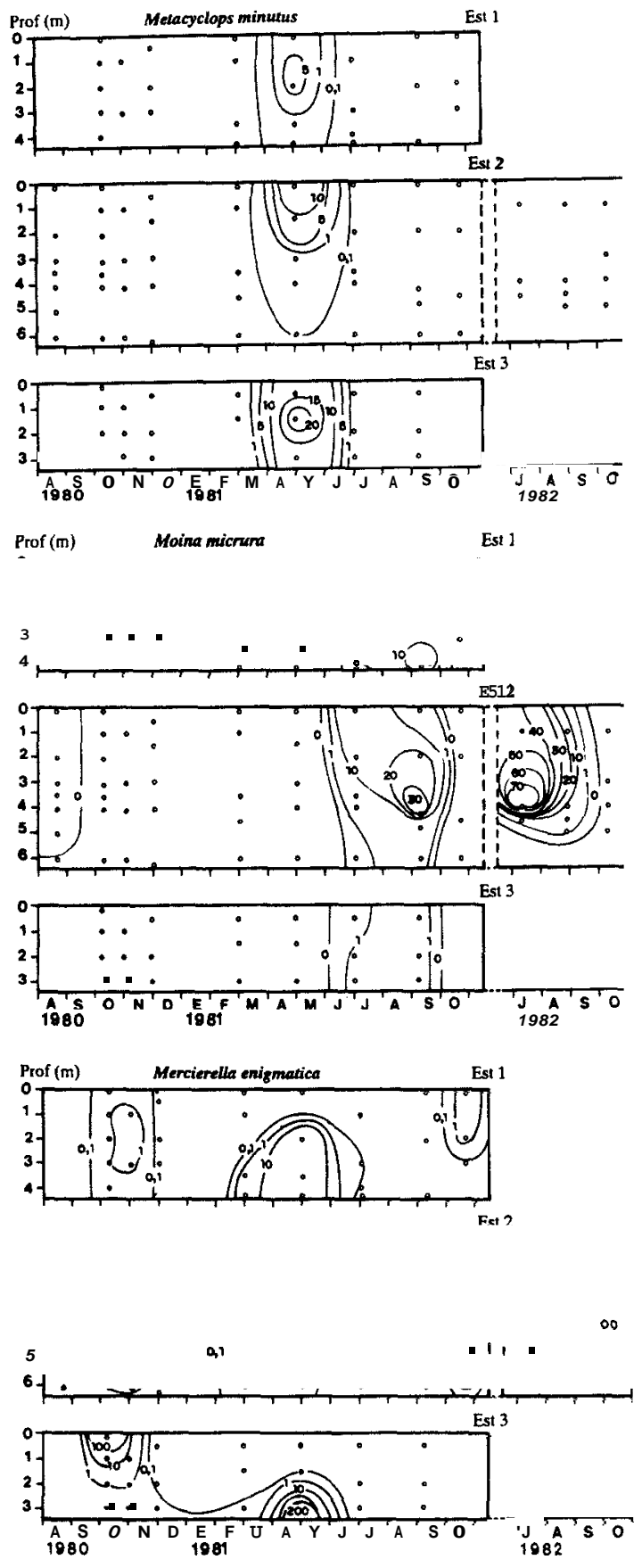

Figura 3. Distribución de la densidad de población (ind/l) en funcion del tiempo y la profundidad, de Metacyclops minutus (arriba), Moina micrura (centro) y larvas de Ficopotamus enigmaticus (Mercierella enigmatica) (abajo) en las tres estaciones de muestreo (Est. 1, Est.2 y Est.3). Distribution of densities (ind/) according to time and depth of species Metacyclops minutus (top), Moina micrura (middle)and Ficopotamus enigmaticus larvae (Mercierella enigmatica) (bottom)in the three sampling points (Est.1, Est.2 y Est.3). en las estaciones 2 y 3 , siempre a una conductividad no superior a $1.1 \mathrm{mS} / \mathrm{cm}$. En otoiio también aparecieron escasos nauplios de cirripedos, en las estaciones 1 y 2 , con una conductividad de 26 a $47.6 \mathrm{mS} / \mathrm{cm}$ (Tabla 1$)$.

\section{Poliquetos}

En los fondos próximos a la desembocadura (estación 1) existian colonias del poliqueto Ficopotamus enigmaticus (Fauvel, 1923), descrito por este autor como Mercierella enigmatica, designándose con este ultimo nombre en muchos trabajos. Parece que las larvas, de vida planctbnica, eran arrastradas al interior de la laguna por las corrientes de agua marina que penetraba en profundidad, sobre todo en el semestre frío de mediados de otoño a mediados de primavera, ya que se encontraban en grandes concentraciones en el extremo final de la cola de la laguna. En el plancton fueron encontradas con mayor abundancia en este periodo, pero también en el resto del ciclo anual, $\mathbf{y}$ preferentemente en las estaciones 2 y 3 (Fig. 3). En mayo de 1981 se observó en la estación 3 un máximo en profundidad de 235 larvas/l, siendo la temperatura del agua de $17^{\circ} \mathrm{C}$ y la conductividad de $30.7 \mathrm{mS} / \mathrm{cm}$.
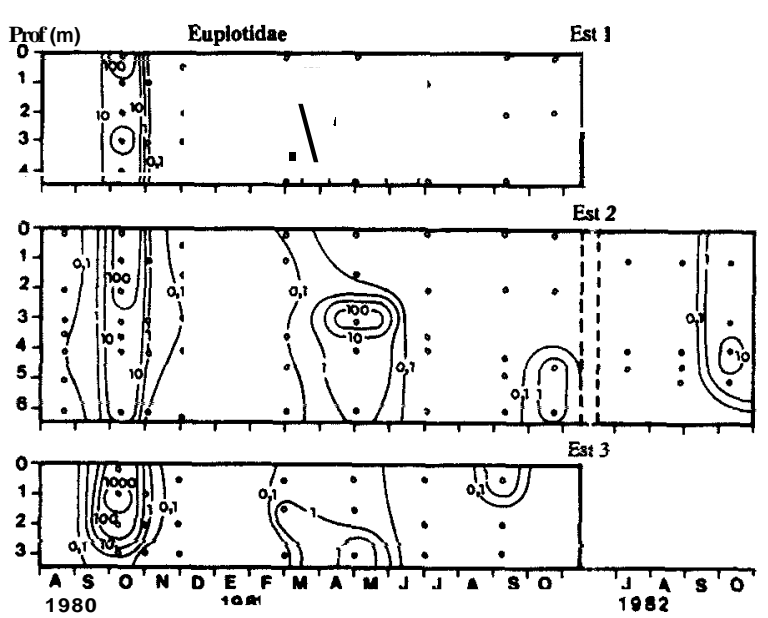

Figura 4. Distribución de la densidad de poblacion (ind/l) en función del tiempo y la profundidad, de los ciliados de la familia Euplotidae (Euplotes sp) en las tres estaciones de muestreo (Est.1, Est.2 y Est.3). Distribution of densities (indn) according to time and depth of ciliates Euplotidae (Euplotes $s p$ ) in the three sampling points (Est.I, Est.2 y Est.3). 


\section{Nematodos}

Se han encontrado nematodos en bajas densidades, generalmente inferiores a 1 ind $/ 1$, en numerosas muestras repartidas en todo el periodo de estudio. Aparecieron con mayor frecuencia en la estacion 3 y en otoiio (noviembre de 1981), observandose densidades de hasta $1 \mathrm{ind} / \mathrm{l}$, cuando la temperatura del agua era de $9^{\circ} \mathrm{C}$ y la conductividad de $1.3 \mathrm{mS} / \mathrm{cm}$ (Tabla 1 ).

\section{Protozoos}

- Género Euplotes. Estos ciliados planctonicos distribuidos por aguas marinas y dulces (Corliss, 1979), eran muy abundantes en el Estany (Fig. 4). Las máximas densidades se observaron en octubre de 1980: 1650 ind/l en la estación 3. En mayo de 1981 se observaron densidades de $170 \mathrm{ind} / \mathrm{l}$ en la estación 2 , a $16^{\circ} \mathrm{Cy}$ conductividad de $30.9 \mathrm{mS} / \mathrm{cm}$.

- Orden Tintinnidu. Se detectaron en invierno, en las tres estaciones de muestreo y en capas de elevada salinidad, a más de $3 \mathrm{~m}$ de profundidad. En la estación 2 se observaron densidades de 5 ind/l, con una temperatura de $13^{\circ} \mathrm{C}$ y conductividad de $41.9 \mathrm{mS} / \mathrm{cm}$.

- Familia Hulteriidue. Estos ciliados aparecieron durante todo el periodo de estudio. Las ma- yores densidades ( $80 \mathrm{ind} / \mathrm{l})$ se observaron en julio de 1982 en la estacion 2 , con temperatura de $22^{\circ} \mathrm{C}$ y conductividad de $43 \mathrm{mS} / \mathrm{cm}$.

- GCnero Arcella. Fueron encontrados ejemplares a lo largo de todo el periodo de estudio, aunque generalmente a densidad inferior a 1 ind/l. En la estación 3 aparecieron con mas frecuencia, observandose en septiembre de 1981 densidades de 1 a $2.5 \mathrm{ind} / \mathrm{l}$, con una temperatura en la columna de agua de $26^{\circ} \mathrm{C}$ y conductividad inferior a la que aparecian otros protozoos, 1.2 $\mathrm{mS} / \mathrm{cm}$.

\section{Densidad y Biomasa}

En las tablas 2 y 3 se indica la densidad y biomasa medias para la columna de agua (desde la superficie hata $4.5 \mathrm{~m}$ en la Est. 1,6 m en la Est. 2 y $3.5 \mathrm{~m}$ en la 3 ) de los distintos grupos zooplanctónicos a lo largo del periodo de estudio. En los meses en que se dispone de muestras en las tres estaciones, se aprecia que, en general, copépodos, protozoos y larvas de poliquetos tendieron a ser más abundantes en la estación 3. Los cladóceros aparecieron con mayor densidad en la estación 2. Todos los grupos alcanzaron sus mayores densidades en verano o principios de otoiio.

Tabla 2. Ndmero medio de individuos en la columna de agua de cada gmpo zooplanctdnico en las diferentes estaciones de muestreo y a lo largo del periodo de estudio (ind/1). Mean number of individuals in the water column in each zooplanktonic group at the different sampling stations throughout the study period (ind/l).

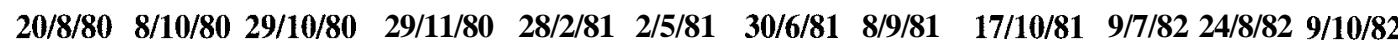

\begin{tabular}{|c|c|c|c|c|c|c|c|c|c|c|c|c|}
\hline \multicolumn{13}{|l|}{ PUNTO 1} \\
\hline Copépodos & & 9.4 & 11.2 & 3.2 & 3.1 & 26.1 & 137.3 & 66.3 & 199.8 & & & \\
\hline Cladóceros & & 0 & 0 & 0 & 0 & 0.1 & 1.3 & 8.3 & 0 & & & \\
\hline Protozoos & - & 75.8 & 0.4 & 0.3 & 0.9 & 14.5 & 0.2 & 0 & 0.3 & & & \\
\hline Poliquetos & & 0.7 & 3.9 & 0.1 & 3.0 & 57.2 & 0.1 & 0.1 & 0.9 & & & \\
\hline \multicolumn{13}{|l|}{ PUNTO 2} \\
\hline Copépodos & 23.1 & 13.5 & 5.5 & 8.2 & 3.1 & 19.8 & 130.5 & 56.6 & 227.1 & 50.3 & 10.4 & 32.6 \\
\hline Claddceros & 0.1 & 0 & 0 & 0 & 0 & 0.1 & 7.8 & 17.6 & 0 & 30.6 & 8.0 & 0 \\
\hline Protozoos & 0.7 & 240.5 & 1.9 & 0.5 & 3.0 & 21.1 & 0.1 & 0 & 0.9 & 23.8 & 0 & 10.5 \\
\hline Poliquetos & 0.1 & 0.8 & 2.6 & 0.1 & 4.5 & 26.2 & 0.1 & 0.1 & 3.8 & 0 & 0 & 90.6 \\
\hline \multicolumn{13}{|l|}{ PUNTO 3} \\
\hline Copépodos & - & 21.1 & 16.2 & 22.6 & 2.7 & 30.9 & 237.2 & 54.5 & & & & \\
\hline Claddceros & & 0 & 0 & 0.1 & 0 & 0.3 & 1.5 & 1.6 & & & & \\
\hline Protozoos & - & 631.9 & 1.7 & 0.7 & 1.4 & 9.7 & 0.2 & 2.3 & & & & \\
\hline Poliquetos & - & 23.3 & 1.1 & 0.5 & 0.3 & 85.1 & 0.1 & 0.3 & & & & \\
\hline
\end{tabular}


Tabla 3. Biomasa media en $\mathrm{mg} / \mathrm{m}^{3}$ en la columna de agua de los grupos zooplanctónicos indicados. a lo largo del periodo de estudio, en las tres estaciones de muestreo. Meun biomass (in $\mathrm{mg}_{\mathrm{g}} / \mathrm{m}^{3}$ ) for the water column of the zooplanktonic groups, throughout the study ut the three sampling stations.

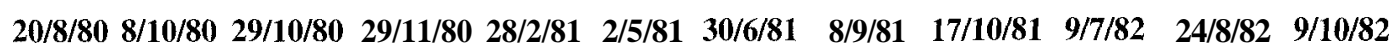

\begin{tabular}{|c|c|c|c|c|c|c|c|c|c|c|c|c|}
\hline \multicolumn{13}{|l|}{ Estación 1} \\
\hline Copépodos & - & 64.6 & 53.6 & 3.3 & - & 331.4 & 829.2 & 118.2 & 736.4 & & & \\
\hline Claddceros & & 0 & 0 & 0 & - & 0.3 & 26.4 & 345.8 & 0 & & & \\
\hline Protozoos & - & 10.2 & 0.1 & 0.1 & - & 1.0 & 0.1 & 0 & 0 & & & \\
\hline Poliquetos & - & 1.2 & 46.5 & $0 . \mathrm{I}$ & - & 593.8 & 0.9 & 0.1 & 6.1 & & & \\
\hline \multicolumn{13}{|l|}{ Estacion 2} \\
\hline Copepodos & 37.9 & 81.0 & 63.8 & 32.7 & - & 343.5 & 799.9 & 228.6 & 3255.7 & 886.9 & 52.0 & 163.1 \\
\hline Cladóceros & 3.3 & 0 & 0 & 0 & - & 0.2 & 155.2 & 731.2 & 0 & 1214.0 & 246.6 & 0 \\
\hline Protozoos & 0.1 & 32.3 & 0.2 & 0.1 & - & 2.6 & 0.1 & 0 & 0.1 & 0.7 & 0 & 1.4 \\
\hline Poliquetos & 0.1 & 1.4 & 32.2 & 0.1 & - & 271.8 & 0.1 & 0.1 & 25.9 & 0 & 0 & 109.5 \\
\hline \multicolumn{13}{|l|}{ Estación 3} \\
\hline Copépodos & - & 70.9 & 270.1 & 410.5 & - & 357.3 & 467.9 & 40.2 & & & & \\
\hline Cladoceros & & 0 & 0 & 0.3 & $=$ & 2.7 & 28.9 & 66.9 & & & & \\
\hline Protozoos & - & 39.8 & 14.4 & 0.8 & - & 1.2 & 0.1 & 0.2 & & & & \\
\hline Poliquetos & - & 84.9 & 0.2 & 0.1 & - & 876.6 & 0.1 & 0.3 & & & & \\
\hline
\end{tabular}

En cambio los valores minimos se registraron a finales de otoño (noviembre de 1980) y en invierno (febrero de 1981).Las mayores densidades las alcanzaron protozoos y copépodos: $631 \mathrm{ind} / 1$ (octubre 1980) y $227 \mathrm{ind} / \mathrm{l}$ (octubre 1981), respectivamente. Cladoceros y larvas de poliquetos registraron densidades algo menores: $30 \mathrm{ind} / \mathrm{l}$ (julio 1982) y $90 \mathrm{ind} / \mathrm{l}$ (octubre 1982), respectivamente.

Los valores maximos de biomasa coincidieron con los valores maximos de densidad en cada grupo, excepto en el caso de los poliquetos. Estos alcanzaron el valor máximo $\left(876 \mathrm{mg} / \mathrm{m}^{3}\right)$ en mayo de 1981 en la estacion 3, debido al mayor tamaño de las larvas ese mes. Los copépodos y cladóceros alcanzaron unos valores maximos de 4.2 y $1.2 \mathrm{~g} / \mathrm{m}^{3}$, respectivamente, muy por encima de los máximos de los otros grupos. En el caso de los protozoos la biomasa máxima fue $39 \mathrm{mg} / \mathrm{m}^{3}$.

La figura 5 muestra la contribución relativa de cada grupo zooplanctonico (incluyendo los rotiferos) a la densidad y biomasa total. Puede apreciarse que aunque numericamente los rotíferos solían ser el grupo dominante, en terminos de biomasa eran los copepodos los mas importantes. Los cladóceros cuando aparecian contribuian de manera notable a la biomasa, debido a
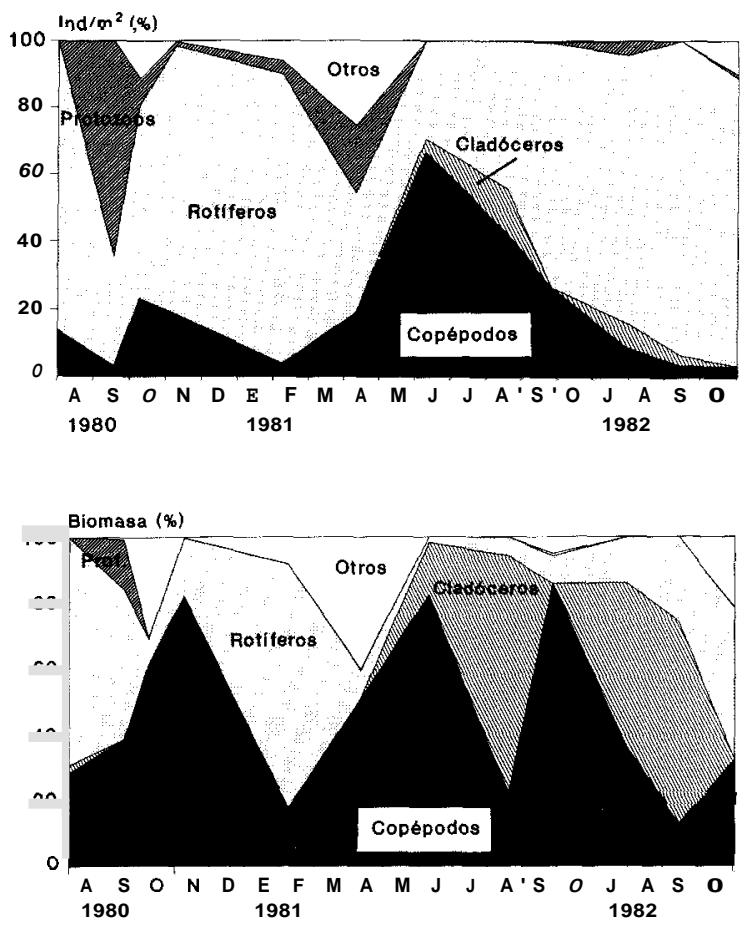

Figura 5. Contribución realtiva de cada gmpo zooplanctónico al número total de individuos (arriba) y a la biomasa (abajo) en toda la columna de agua de la estación 2. Relative. ontribution of each zooplanktonic group to the total number of individuals (top) and to the biomass (bottom) in all the water column at station 2. 
Tabla 4. Coeficientes de correlacion estadísticamente significativos $(p<0.05)$, tras la corrección de Bonferroni (Rice, 1988), entre las especies que se indican y la temperatura y conductividad. Con un asterisco se señalan los coeficientes altamente significativos $(\mathrm{p}<0.01)$. Statistically significant correlation coefficients $(p<0.05)$, after Bonferroni correction (Rice. 1988), between density of the species indicated or temperature and conductivity. Highly significant coefficients $(p<0.01)$ are indicated with an asterisk.

\begin{tabular}{lcc}
\hline & Temp. & Conduc. \\
\hline Calanipeda aquae-dulcis & $0.49^{\prime \prime}$ & \\
Acanthocyclops robustus & $0.35^{*}$ & $-0.35^{\prime \prime}$ \\
Metacyclops minutus & & \\
Moina rnicrura & $0.52^{\prime \prime}$ & $-0.28^{\prime \prime}$ \\
Mercierella enigmatica & & $0.43^{\prime \prime}$ \\
Euplotes sp. & & 0.20 \\
\hline
\end{tabular}

su tamaiio. Los protozoos fueron numericamente importantes al final del verano de 1980, pero nunca tuvieron repercusión destacable en la biomasa. Las larvas de poliqueto adquirian mayor importancia relativa en otoiio y primavera.

\section{Distribución vertical y ciclo anual}

En la Tabla 4 se muestran las correlaciones de las especies más abundantes con la temperatura y la conductividad. En el ciclo anual la conductividad aumentaba en el semestre frio de mediados de otofio a mediados de primavera y en el perfil vertical aumentaba con la profundidad, de modo que las especies que presentan correlaciones positivas con este parámetro, es porque eran más abundantes en el periodo frio o porque en la columna de agua se situaban sobre la haloclina.

En verano las especies caracteristicas eran Acanthocyclops robustus y Moina micrura, distribuidas entre las capas superficiales y la haloclina (correlacion negativa con la conductividad). También estaba presente Calanipeda aquae-dulcis, especie que alcanzaba su mayor desarrollo en otofio, en las capas de la haloclina. En la misma estacion y en las capas situadas sobre la haloclina (correlación positiva con la conductividad) alcanzaban sus máximos las larvas del poliqueto F. enigmaticus y los ciliados del genero Euplotes.
En invierno habia pocas especies en el plancton y aparte de la perenne $C$. aquae-dulcis merece destacarse la aparicion de los copepodos marinos Acartia clausi y Oithona nana. En primavera destacaba la aparición del copépodo Metacyclops minutus, en aguas superficiales, y los nuevos máximos de F. enigmaticus y ciliados Euplotes sp. sobre la haloclina.

\section{DISCUSIÓN}

La entrada periódica de agua de mar en el Estany de Cullera y la presencia constante de una capa salada en el fondo, favorecia la aparición de especies eurihalinas o marinas. Entre éstas ultimas cabe citar los copépodos Euterpina acutifrons, Acartia clausi, A. grani, Oithona nana, larvas del poliqueto $F$. enigmaticus, ciliados del género Euplotes y nauplios de cirripedos. Es de destacar el transporte a 10 largo de toda la laguna, mas de 2 $\mathrm{Km}$ aguas arriba, de todos estos organismos, encontrándose en concentraciones incluso mayores en la estación 3, en el extremo proximo al rio.

Merece destacarse la presencia del copépodo eurihalino y euritermo Calanipeda aquae-dulcis, de distribución circum-mediterranea. En España esta especie ha sido encontrada en aguas litorales, pero no en el interior peninsular. Se ha citado en humedales de Catalufia y Menorca (Margalef, 1953; Chinchilla \& Comin, 1977; Forés et al., 1986), estuario del río Guadalquivir (Guisande $e t$ al., 1986) y en varios humedales levantinos (Oltra \& Armengol-Diaz, 1999). La especie Acanthocyclops robustus ademhs de ser frecuente en lagunas litorales (Oltra \& Miracle, 1992), también se encuentra en embalses, muchas veces como especie dominante (Armengol, 1978, 1980).

Se ha confirmado el carácter termófilo de la especie Moina micrura, la cual solo ha aparecido en la estacion chlida. Su presencia en el Estany en aguas relativamente saladas (densidades de hasta 16 ind/l a salinidades de 2I \%o), asi como en lagunas costeras del Delta del Ebro a salinidades de 5 $\%$, contrasta con la baja salinidad de los embalses y lagunas interiores donde esta presente (Armengol, 1978; Alonso, 1985). Todas las 
demás especies de cladóceros acompafiantes (Tabla 1), han sido encontradas con anterioridad en lagunas costeras mediterráneas (Arévalo, 1916; Blanco, 1976; Forés et al., 1986; Oltra \& Miracle, 1992), lagunas interiores (Alonso, 1985) y, excepto Leydigia acanthocercoides, también en embalses (Armengol, 1978). Son especies de distribución cosmopolita y generalmente de hábitos bénticos (Margalef, 1953; Flossner, 1972; Alonso, 1985).

El poliqueto Ficopotamus enigmaticus es originario de las regiones australes subtropicales pero llegado al Mediterraneo a traves del Canal de Suez, ha colonizado recientemente con éxito las zonas eutróficas del litoral mediterrrineo. En España ha sido citada en el Grao de Gandia (Rioja, 1931), el Mar Menor (Garcia Carrascosa, 1979) y las Albuferas de Mallorca y Menorca (Martinez-Taberner et al., 1993).

A pesar de que las muestras de zooplancton se filtraron por mallas de $45 \mu \mathrm{m}$, por tanto no adecuadas para la recolección de protozoos, en muchas ocasiones se observaron elevadas densidades de ciliados, por 10 que cabe pensar que su abundancia en el plancton podria ser elevada. La mayoría eran del género Euplotes, perteneciente a la familia Euplotidae, ampliamente distribuida por todo el mundo, con preferencia en aguas marinas (Corliss, 1979).

Los crustriceos del zooplancton del periodo estival de influencia continental son carcterísticos de las lagunas litorales eutróficas que reciben aguas de excedentes de cultivo, en gran parte de arrozales. La presencia de $M$. minutus, A. robustus y $M$. micrura, va ligada a estos ambientes y la dominancia de estos organismos depende de la mayor afluencia de dichas aguas, por ello se favorecen estas especies en los años sin restricciones de regadio. Ciclos similares con periodos de mayor o menor influencia marina durante otofio invierno y primavera suceden en las lagunas del Delta del Ebro (Menéndez \& Comin, 1986), observándose una alternancia entre las especies C. aquaedulcis y A. robustus similar a la observada en Cullera y dependiendo la mayor o menor presencia de A. robustus de la diferente importancia de la afluencia de las aguas conti- nentales en la distintas lagunas o canales. En lagunas como la Albufera de Menorca (Pretus, 1989) no sometidas a un regimen dominado por los regadios, la salinidad es más alta y con un ciclo anual diferente, incrementandose en general en verano, de manera que la composición específica de los crustaceos planctónicos es totalmente diferente.

\section{AGRADECIMIENTOS}

Este estudio estaba integrado en un proyecto de investigación $\left(\mathrm{n}^{\circ} 3778 / 79\right)$ financiado por la CAICYT. Agradecemos a Eduardo Vicente, Carles Abellà y Ricard Guerrero la colaboración en la toma de muestras y medidas de variables in situ.

\section{BIBLIOGRAFÍA}

ALFONSO, M.T. \& M.R. MIRACLE. 1990. Distribución espacial de las comunidades zooplanctónicas de la Albufera de Valencia. Scientia gerundensis, 16: 11-25.

ALFONSO, M.T. \& M.R. MIRACLE. 1996. Estudio de las comunidades zooplanctdnicas de los ecosistemas acuáticos del Parque Natural de la Albufera de Valencia. Tesis Doctoral, Universidad de Valencia. $310 \mathrm{pp}$.

ALONSO, M. 1985. Las lagunas de la España peninsular: taxonomia, ecología y distribución de los cladóceros. Tesis Doctoral. Universidad de Barcelona. $79.5 \mathrm{pp}$.

AREVALO, C. 1916. Introducción al estudio de los cladoceros del plancton de la Albufera de Valencia. Anales del Instituto General y Técnico de Valencia. Vol. I, (1), $6.5 \mathrm{pp}$.

ARMENGOL, J. 1978. Los crustaceos del plancton de los embalses espaiioles. Oecologia acuatica, 3: 396.

ARMENGOL, J. 1980. Colonización de los embalses espafioles por crustáceos planctónicos y evolución de la estructura de sus comunidades. Oecologia aquatica, 4: 45-70.

BIANCHI, C.N. 1981. Policheti Serpuloidei. Guide per il riconoscimento delle specie animali delle acque lagunari e costiere italiane. Consiglio Nazionale delle Ricerche AQ/1/96. 5, 187 pp. 
BLANCO, C. 1976. Estudio de la contaminación de la Albufera de Valenciay de los efectos de dicha contaminacion sobre la fauna y flora del lago. Tesis Doctoral, Universidad de Valencia, 193 pp.

CARRILLO, P., L. CRUZ-PIZARRO, R. MORALES \& P. SANCHEZ-CASTILLO. 1987. Cambios estacionales en las comunidades de fitoplancton y de zooplancton de la Albufera de Adra. Limnetica, 3: 243-254.

CHAMPEAU, A. 1970. Recherches sur l'écologie et l'adaptation a la vie lutente des cope'podes des eaux temporaires provençales et corses. Tesis Doctoral, Universidad de Marsella.

CHINCHILLA, M. \& F.A. COMIN. 1977. Contribucio al coneixement dels crustacis del Delta de 1'Ebre. Treb. Inst. Cat. Hist. Nat., 8: 119-144.

CORLISS, J.O. 1979. The ciliated Protozoa. Characterization, Classification and Guide to the Literature. 455 pp.

CURDS, C.R. 1982. British and other freshwater ciliated protozoa. Part 1 Ciliophora: Kinetofragminophora. Synopses of the British Fauna, 22. D.M. Kermack \& R.S.K. Barnes (eds.). 387 pp.

DUSSART, B. 1967. Les cope 'podes des eaux continentales d'Europe occidentale. Tome I: Calanoüdes et Harpacticoïdes. Boubée et Cie Ed. Collection "Faunes et flores actuelles". Paris. 500 pp.

DUSSART, B. 1969. Les cope "podes des eaux continentules d'Europe occidentale. Tome II: Cyclopoïdes et Biologie. Boubée et Cie Ed. Collection "Faunes et flores actuelles". Paris. 292 pp.

EDMONDSON, W.T. 1971. Methods for processing samples and developing data. Cap. 2: 127-169.En:. A manual on methods for the assessment of secondary productivity in fresh waters. W.T. Edmondson \& G.G. Winberg (eds.). IBP Handbook no. 17. Oxford: Blackwell.

FAUVEL, P., 1923. Un nouveau serpulien d'eau saumâtre Mercierella n.g. enigmatica n. sp.. Bull. Soc. Zool. Fr. 46: 424-430.

FLOSSNER, D. 1972. Krebstiere, Crustacea. Kiemen - und Blattfüsser, Branchiopodu; Fischlause, Branchiura. Tierwelt Deutschlands, 60: 1-501.

FORÉS, E., M. MENÉNDEZ \& F.A. COMÍN. 1986. Contribución al conocimiento de crustáceos y rotiferos del Delta del Ebro. Misc. Zool., 10: 105111.

FRYER, G. 1978. Free-living stages of freshwater parasitic copepoda. In: Das zooplankton der binnengewässer, 2 Teil: 1-343 (die Binnengewässer 26, 2 Teil). Schweizerbart, Stuttgart.
GARCIA CARRASCOSA, A.M. 1979. Contribucion al conocimiento del bentos del Mar Menor: poblamientos bentónicos de las islas Perdiguera, Redonda y del Sujeto. Estudio descriptivo y cartografia bionomica. Primer simposio de Bentos, San Sebastian, 9-11 Abril, 1979.

GUISANDE C., T. LOPEZ \& J. TOJA. 1986. Zooplancton del estuario del río Guadalquivir. II Simposio del Agua en Andaluciu, Vol I: 361-372.

KIEFER, F. 1978. Freilebende Copepoda. In:Das Zooplankton der Binnengewusser (2):

KUDO, R.R. 1976. Protozoología. C.E.C.S.A. México. $905 \mathrm{pp}$.

MARGALEF, R. 19.53. Los crustáceos de las uguas continentules ibéricas. Biología de las aguas continentales X. Min. Agricultura. Inst. For. Inv. Exp., Madrid, 243 pp.

MARINEZ-TABERNER, A. V. FORTEZA \& J.J. FORNÓS. 1993. Colonization, structure and growth of Ficopotamus enigmaticus cf. Ten Hove \& Weerdenburg (Polychaeta, Serpulidae) in the Albufera of Menorca, Balearic Islands. Verh. Internat. Verein. Limnol., 2.5: 1031-1034.

MENENDEZ, M. \& F.A. COMIN. 1986. Variacion estacional del zooplancton en las lagunas costeras del Delta del Ebro (N.E. España). Oecologia Aquatica, 8: 47-60.

MIRACLE, M.R. \& E. VICENTE. 1983. Vertical distribution and rotifer concentrations in the chemocline of meromictic lakes. Hydrobiologia, 104:259-267.

MIRACLE, M.R. \& E. VICENTE. 1985. Phytoplankton and photosynthetic sulphur bacteria production in the meromictic coastal lagoon of Cullera (Valencia, Spain). Verh. Internat. Verein. Limnol., 22: 2214-2220.

OLTRA, R. 1993. Estudio del zooplancton de dos lagunas litorales mediterraneas: El Estany de Cullera y la Albufera de Valencia. Tesis Doctoral, Universidad de Valencia. 437 pp.

OLTRA, R. \& X. ARMENGOL-DIAZ 1999. Limnología de los humedales valencianos suceptibles de albergar samaruc y fartet: (11) zooplancton. En: Monografía sobre los peces ciprinodóntidos ibéricosfartet y samaruc. Generalitat Valenciana (ed.). Colección Biodiversidad no 5 : 79-97

OLTRA, R. \& M.R. MIRACLE 1992. Seasonal succession of zooplankton populations in the hypertrophic lagoon Albufera of Valencia (Spain). Arch. Hydrobiol., 124: 187-204.

OLTRA, R. \& M.R. MIRACLE, 2000a. Variación espacio-temporal de las poblaciones de rotiferos de la 
laguna meromictica Estany de Cullera (Valencia). Limnetica, 19:39-52.

OLTRA, R. \& M.R. MIRACLE, 2000b. Estructura de la comunidad zooplanctónica de la laguna meromictica Estany de Cullera (Valencia). Limnetica, 19:67-82.

PONT, D. 1977. Structure et évolution saisonnière des populationde copépodes, cladocères et ostracodes des rizières de Camargue. Annls. Limnol., 13: 15-28.

PRETUS, J.L. 1989. Limnologia de la Albufera de Menorca (Menorca, España). Limnetica, 5: 69-81.

PRETUS, J.L., J. DE MANUEL \& L. CARDONA. 1992. Temporal heterogeneity, zooplankton composition and fish food supply in the Albufera of Minorca, a highly fluctuant environment. Bulletin de 1'Institut océanographique, Monaco, $\mathrm{n}^{\circ}$ spécial 11: 179-188.

RICE, W.R. 1988. Analyzing tables of statistical tests. Evolution, 43 (1): 223-225.
RIOJA, E. 1931. Estudio de los poliquetos de la peninsula Iberica. Mem. Acad. Cienc. Madrid, ser. Cien. Nat., 2:1-471.

ROJO, C. \& M.R. MIRACLE. 1984. Fluctuación estacional de las poblaciones fitoplanctonicas del Estany de Cullera (Valencia). Anales de Biología, 2 (Sec. Esp., 2): 161-168.

ROJO, C. \& M.R. MIRACLE 1989. Phytoplankton fluctuations during an annual cycle in the coastal lagoon of Cullera (Spain). Int. Revue ges. Hydrobiol., 74: 179-194.

ROJO, C., M.R. MIRACLE \& M. SERRA. 1986. Interrelacion entre las especies de microalgas halladas en el Estany de Cullera (Valencia). Limnetica, 2: 35-40.

RUTTNER-KOLISKO, A. 1977. Suggestions for biomass calculations of plankton rotifers. Arch. Hydrobiol. Beih. Ergebn. Limnol., 8: 71-76. 